\title{
A Phase I/II Study of NAC with Docetaxel, Cisplatin, and S-1 for Stage III Gastric Cancer
}

\author{
YUSAKU TANAKA ${ }^{1}$, CHIKARA KUNISAKI $^{1}$, YUSUKE IZUMISAWA ${ }^{1}$, HIROCHIKA MAKINO $^{1}$, \\ JUN KIMURA ${ }^{1}$, SHO SATO ${ }^{1}$, HIROSHI MIYAMOTO ${ }^{1}$, TAKASHI KOSAKA ${ }^{1}$, HIDETAKA A. ONO ${ }^{1}$, \\ MASAZUMI TAKAHASHI ${ }^{2}$, KEI SATO $^{3}$, HIROTOSHI AKIYAMA ${ }^{3}$ and ITARU ENDO ${ }^{3}$ \\ ${ }^{1}$ Department of Surgery, Gastroenterological Center, Yokohama City University, Yokohama, Japan; \\ ${ }^{2}$ Department of Surgery, Yokohama Municipal Hospital, Yokohama, Japan; \\ ${ }^{3}$ Department of Gastroenterological Surgery, Graduate School of Medicine, \\ Yokohama City University, Yokohama, Japan
}

\begin{abstract}
Background/Aim: The aim of this phase I/II study was to determine the safety, and efficacy of combination of neoadjuvant chemotherapy (NAC) with biweekly docetaxel, cisplatin, and S-1 (DCS) in stage III gastric cancer patients. Patients and Methods: In the phase I study, SI was administered at doses of $80 \mathrm{mg} /$ day to $120 \mathrm{mg} /$ day depending on the body surface area and docetaxel was administered at $20 \mathrm{mg} / \mathrm{m}^{2}$, whereas cisplatin was initially administered at 25 $\mathrm{mg} / \mathrm{m}^{2}$ and was escalated by $5 \mathrm{mg} / \mathrm{m}^{2}$ up to $50 \mathrm{mg} / \mathrm{m}^{2}$. In the phase II study, safety and therapeutic efficacy of DCS were evaluated using the recommended dose of cisplatin. Results. In phase I, 21 patients were enrolled. In level II, perforation of gastric cancer occurred in one case although no dose limiting toxicities (DLTS) were noted in level III-VI. Recommended dose for cisplatin was $50 \mathrm{mg} / \mathrm{m}^{2} /$ day. In phase II, among 47 patients, 14 experienced grade 3/4 adverse events. Clinically, response rate was $66.7 \%$ and disease control rate was $97.9 \%$. The curative $(R 0)$ resection rate was 95.7\%. Pathological response rate was 53.3\%. Three-year overall survival and relapse-free survival rates were $78.5 \%$ and $65.3 \%$, respectively. Conclusion: Biweekly DCS as NAC was efficient, safe, and acceptable; however, long-term survival should be evaluated to confirm the efficacy of biweekly DCS for stage III gastric cancer patients.
\end{abstract}

Correspondence to: Chikara Kunisaki, Department of Surgery, Gastroenterological Center, Yokohama City University, 4-57, Urafune-cho, Minami-ku, Yokohama, 232-0024, Japan. Tel: +81 452615656, Fax: +81 452619492, e-mail: s0714@med.yokohamacu.ac.jp

Key Words: Docetaxel, cisplatin, S-1, gastric cancer, neoadjuvant chemotherapy.
Surgery remains one of the most important treatment options for gastric cancer, which is still one of the most common malignancies globally, despite declining incidence rates (1). Although curative (R0) resection is associated with significant cure rates, $<50 \%$ of locally advanced gastric cancer patients can successfully undergo $\mathrm{R} 0$ resection even with aggressive surgery (2). Moreover, in a large proportion of patients with advanced gastric cancer, disease recurs after curative resection; consequently, the survival rates remain unsatisfactory even after super-extended lymph node dissection (3).

A recent Japanese large-scale phase III trial by the adjuvant chemotherapy of S-1 (tegafur/gimeracil/oteracil) for gastric cancer patients reported the superiority of S-1 as adjuvant chemotherapy over surgery alone after D2 lymph node dissection in stage II/III patients (4). However, the subgroup analysis of stage IIIB gastric cancer patients showed that overall survival (OS) and recurrence-free survival rates did not differ between the S-1 and surgeryalone groups (5). Therefore, more effective chemotherapy regimens are necessary to improve prognosis in stage III gastric cancer patients.

Neoadjuvant chemotherapy (NAC) has been proposed to have certain benefits compared with adjuvant chemotherapy, including downstaging that might allow for subsequent R0 resection, treatment of micrometastases, and evaluation of the susceptibility and tolerability to chemotherapeutic agents (6, 7). Therefore, the development of effective chemotherapeutic regimens is important to improve the efficacy of NAC.

A recent phase II trial evaluated the efficacy of a combination regimen containing docetaxel, cisplatin, and S1 (DCS) in unresectable or recurrent gastric cancer patients and revealed that the response rate was high (8). However, this phase II trial, as well as other phase I studies using DCS regimens reported that grade 3 or 4 neutropenia, nausea and vomiting frequencies were higher with cisplatin doses of 
60-70 mg/m² (9-11). Additionally, patients should be sufficiently hydrated to avoid cisplatin-induced renal damage during DCS regimen. Therefore, it is necessary to safely offer treatment with these DCS regimens in the hospital (12).

Although chemotherapy for gastric cancer is often administered on an inpatient basis, many patients request outpatient chemotherapy to minimize disruption of their daily life. Although DCS-containing combination therapies were previously reported to be beneficial in advanced gastric cancer, they were also reported to be closely associated with grade $\geq 3$ adverse events and prolonged hospital stays. These findings highlight the necessity to develop improved triplet therapies, including DCS-containing therapies, that are not associated with severe adverse events and can be administered at an outpatient clinic.

Therefore, a phase I/II trial was conducted using combination NAC with biweekly DCS administration by escalating cisplatin dose at an outpatient setting to assess its efficacy on therapeutic outcomes in clinical stage III gastric cancer patients

\section{Patients and Methods}

Patient eligibility. The present study was conducted at the Department of Surgery, Gastroenterological Center, Yokohama City University in Yokohama, Japan, and its related institutions. The eligibility criteria were as follows: (i) histologically proven gastric adenocarcinoma, (ii) stage III gastric cancer (according to the Japanese Classification of Gastric Carcinoma 14th edition) (13) without peritoneal metastasis confirmed by staging laparoscopy, (iii) age of 20-75 years, (iv) Eastern Clinical Oncology Group (ECOG) performance status of 0 or 1 , (v) no prior chemotherapy or radiotherapy, (vi) adequate bone marrow function (white blood cell count $\geq 3000$ or $\leq 12000$ per $\mathrm{mm}^{3}$; neutrophil count $\geq 1500$ per $\mathrm{mm}^{3}$; hemoglobin $\geq 9.0 \mathrm{~g} / \mathrm{dl}$; and platelet count $\geq 100,000$ per $\mathrm{mm}^{3}$ ), (vii) adequate hepatic function (serum total bilirubin $\leq 1.5 \mathrm{mg} / \mathrm{dl}$; aspartate aminotransferase and alanine aminotransferase $\leq 2.5$ times the upper normal limit), (viii) adequate renal function (serum creatinine $<1.5 \mathrm{mg} / \mathrm{dl}$; blood urea nitrogen, equal or less than the upper limit of normal), (ix) no other malignancies, and (x) written informed consent. Exclusion criteria were as follows: (i) active infection; (ii) mental disorder; (iii) interstitial pneumonia; (iv) severe comorbidities, such as heart or renal disease; (v) pregnant or lactating women or women of childbearing age unless they were practicing effective contraception.

Administration and dose escalation. S-1 was administered orally twice daily for 1 week according to body surface area (BSA), followed by a drug-free 1-week interval, as follows: BSA $<1.25 \mathrm{~m}^{2}, 80 \mathrm{mg} /$ day; $B S A \geq 1.25 \mathrm{~m}^{2}$ or $<1.50 \mathrm{~m}^{2}, 100 \mathrm{mg} /$ day; BSA $\geq 1.50 \mathrm{~m}^{2}, 120 \mathrm{mg} /$ day. Docetaxel and cisplatin were administered intravenously on days 1 and 15. Docetaxel was administered by intravenous infusion lasting $60 \mathrm{~min}$ at a dose of $20 \mathrm{mg} / \mathrm{m}^{2}$, followed by cisplatin administered as a 120 min intravenous infusion. In phase I of the study, the cisplatin initiation dose was $25 \mathrm{mg} / \mathrm{m}^{2}$ (level I) and was planned to be escalated in $5-\mathrm{mg} / \mathrm{m}^{2}$ increments to $50 \mathrm{mg} / \mathrm{m}^{2}$ at an outpatient clinic setting. The DCS regimen with cisplatin $60-70 \mathrm{mg} / \mathrm{m}^{2}$ has been reported to require a few days of hospital stay for hydration (14). Therefore, maximum cisplatin dose was set to $50 \mathrm{mg} / \mathrm{m}^{2}$ to perform this regimen safely in an outpatient clinic. Dose-limiting toxicities (DLTs) were based on two courses of the DCS regimen (Figure 1).

Treatment with granulocyte colony-stimulating factor was permitted in patients who developed grade 4 neutropenia or grade 3-4 neutropenia with fever. Prophylactic administration of antiemetic therapy, including a 5-HT3 antagonist, a NK1 receptor antagonist, and a corticosteroid, was routinely used to prevent nausea and vomiting due to docetaxel and cisplatin.

Toxicity was graded according to the Common Terminology Criteria for Adverse Events, version 4.0 (15). DLT was defined based on the following: grade 4 leukopenia or neutropenia; grade 3 neutropenia with a fever $\left(\geq 38.0^{\circ} \mathrm{C}\right)$; grade 4 thrombocytopenia; grade 3-4 non-hematological toxicity with the exceptions of nausea, vomiting, and alopecia.

A minimum of three patients were tested for each cisplatin dose level. The dose escalation schedule was as follows. If DLT was not observed in any of the three cases, the dose was increased to the next level. If DLT was observed in one of the three cases, three additional patients were treated at the same dose level. If only one of the six patients experienced DLT, the dose was increased to the next level. However, if two or more of the six patients exhibited DLT, the dose level was defined as the maximum tolerated dose. Dose escalation was continued until the maximum tolerated dose was reached, and the level one step below was set as the recommended dose (RD) for further evaluation in phase II of the study.

Disease evaluation. Tumor response was basically assessed based on the Response Evaluation Criteria in Solid Tumors (RECIST) version 1.1 (16) after completion of the two treatment courses. Response status during the treatment was evaluated by barium meal study, endoscopy, and computed tomography. Response rate was calculated as follows: complete response $(\mathrm{CR})+$ partial response $(\mathrm{PR}) / \mathrm{CR}+\mathrm{PR}+$ stable disease $(\mathrm{SD})+$ progressive disease $(\mathrm{PD})$. Disease control rate was defined as follows: $\mathrm{CR}+\mathrm{PR}+\mathrm{SD}+($ Non$\mathrm{CR} /$ Non-PD) $/ \mathrm{CR}+\mathrm{PR}+\mathrm{SD}+($ Non-CR/Non-PD $)+\mathrm{PD}$.

Surgery. Surgery was performed at least 4 weeks after the end of the second cycle in patients who were determined as candidates for curative resection. The type of surgery was based on the location and extent of the primary lesion. The extent of standard lymph node dissection was D2 lymphadenectomy with R0 surgery. If R0 surgery was impossible, D1 lymphadenectomy was performed (13).

Pathological examination of surgical specimens. All resected specimens were examined by experienced pathologists, and the pathological response to chemotherapy was evaluated according to the criteria of the Japanese Research Society for Gastric Cancer (13). Specimens were graded from 0 to 3 based on the following method: grade 0 , no necrosis or cellular or structural changes observed throughout the lesion; grade 1a, necrosis or disappearance of tumor in < one-third of the entire lesion, or only visible cellular or structural changes; grade $1 \mathrm{~b}$, viable tumor cells remaining in $>$ one-third but $\leq$ two-thirds of the tumor area; grade 2 , necrosis or disappearance of tumor in $\geq$ two-thirds of the entire lesion, in addition to the presence of viable tumor cells; grade 3 , necrosis or fibrotic replacement of the entire lesion, with or without granulomatous changes and the absence of viable tumor cells. In the present study, pathological grade 0 or 1 a cancer patients were 


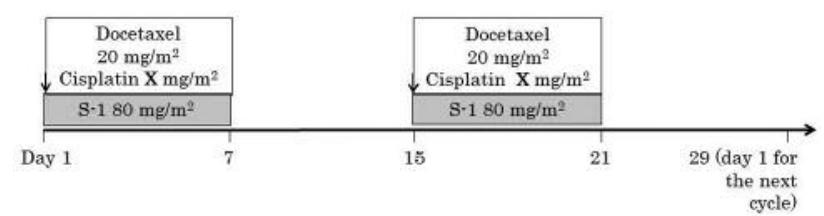

Figure 1. Treatment schedule for the biweekly administration of docetaxel, cisplatin, and S-1.

considered as pathological non-responders, and those with pathological grade $1 \mathrm{~b}, 2$, or 3 cancers were considered as pathological responders, based on classification systems used in previous studies $(17,18)$.

This phase I/II study was approved by the institutional review board of the Yokohama City University, School of Medicine, including the protocol amendment. The study was registered with UMIN-CTR, number UMIN000011625.

Ethical approval. All procedures performed were in accordance with the ethical standards of the institutional and/or national research committee and with the 1964 Helsinki declaration and its later amendments or comparable ethical standards.

Informed consent. Informed consent was obtained from all individual participants included in the study.

Statistical analysis. OS was calculated from the start of the treatment to the date of the last follow-up or death and estimated by the Kaplan-Meier method. Statistical analysis was performed using the SPSS software package version 23.0 (SPSS, Chicago, IL, USA). Based on the Southwest Oncology Group two-stage design, to test the hypothesis with an expected value of $65 \%$ of the response rate for the DCS regimen, a threshold value of $40 \%$, one-sided interim alpha of 0.05 , and one-sided beta of 0.1 , a minimum of 41 patients were required.

\section{Results}

Characteristics of patients in the phase I trial. Characteristics of a total of 21 patients who were enrolled between October 2011 and March 2016 in the phase I trial are presented in Table I. Briefly, patients were predominantly male, and median age was 65 years. Over $80 \%$ of the patients had an ECOG PS score of 0 , and the most frequent tumors were in the upper third of the stomach and were T4 tumors.

Dose escalation schedule. In the phase I trial, none of the patients developed hematological DLTs at any of the dose levels. At dose level II, grade 3 leukopenia and neutropenia were noted, whereas grade 3 or 4 hematological toxicities were not observed at higher dose levels. At dose level II, grade 3 perforation of gastric cancer as DLT was observed in one patient. At all other dose levels, only grade 1 or 2 non-hematological toxicities were observed. In the present
Table I. Characteristics of patients in the phase I trial.

\begin{tabular}{lcc}
\hline Characteristic & No. of patients & $\%$ \\
\hline Total & 21 & \\
Male & 14 & 66.7 \\
Female & 7 & 33.3 \\
Age (years) & & \\
Median & 65 & \\
Range & $45-75$ & \\
ECOG performance status ${ }^{\mathrm{a}}$ & & \\
0 & 17 & 81.0 \\
1 & 4 & 19.0 \\
Histological type & & \\
Differentiated & 12 & 60.0 \\
Undifferentiated & 9 & 40.0 \\
Tumor location & & \\
Upper third & 8 & 38.1 \\
Middle third & 6 & 28.6 \\
Lower third & 4 & 19.0 \\
Entire & 3 & 14.3 \\
Depth of tumor invasionb & & \\
T3 & 5 & 23.8 \\
T4 & 16 & 76.2 \\
Extent of lymph node metastasis ${ }^{\mathrm{b}}$ & & \\
N1 & 7 & 33.3 \\
N2 & 9 & 42.9 \\
N3 & 5 & 23.8 \\
Stage & & \\
IIIA & 9 & 40.0 \\
IIIB & 9 & \\
IIIC & & \\
\hline & & \\
\hline
\end{tabular}

aEastern Cooperative Oncology Group; bAccording to the Japanese Classification of Gastric Carcinoma, 14th edition.

study, the treatment protocol reached dose level VI; therefore, dose level VI with cisplatin at $50 \mathrm{mg} / \mathrm{m}^{2}$ was declared as RD for the phase II part of the study (Table II).

Characteristics of patients in the phase II trial. Characteristics of a total of 47 patients enrolled in the phase II trial were nearly identical to those of the patients in the phase I trial (Table III).

Toxicity in the phase II trial. Toxicity profiles in the phase II trial are shown in Table IV. The most frequent grade 3 adverse events were leukopenia and neutropenia. Febrile neutropenia was observed in two patients, and ten patients $(21.3 \%)$ required dose reduction due to adverse events, including nine patients after the second course and one patient after the third course.

Clinical response to NAC-DCS administration. Based on the study protocol, patients who received two cycles of chemotherapy were evaluated for clinical response in the phase 
Table II. Dose escalation schedule in the phase I trial.

\begin{tabular}{lcccc}
\hline $\begin{array}{c}\text { Dose } \\
\text { level }\end{array}$ & $\begin{array}{c}\mathrm{S}-1 \\
\left(\mathrm{mg} / \mathrm{m}^{2} / \text { day }\right)\end{array}$ & $\begin{array}{c}\text { Docetaxel } \\
\left(\mathrm{mg} / \mathrm{m}^{2}\right)\end{array}$ & $\begin{array}{c}\text { Cisplatin } \\
\left(\mathrm{mg} / \mathrm{m}^{2}\right)\end{array}$ & MTD \\
\hline 1 & 80 & 20 & 25 & $\begin{array}{c}0 / 3 \text { DLT } \\
1 / 6 \text { DLT (n=1, } \\
2\end{array}$ \\
& 80 & 20 & 30 & $\begin{array}{c}\text { gastric perforation }) \\
0 / 3 \text { DLT }\end{array}$ \\
3 & 80 & 20 & 35 & $0 / 3$ DLT \\
4 & 80 & 20 & 40 & $0 / 3$ DLT \\
5 & 80 & 20 & 45 & $0 / 3$ DLT \\
6 & 80 & 20 & 50 & \\
\hline
\end{tabular}

MTD: Maximum tolerable dose; DLT: dose limiting toxicity.

Table III. Characteristics of the patients in the phase II trial.

\begin{tabular}{|c|c|c|}
\hline Characteristic & No. of patients & $\%$ \\
\hline Total & 47 & \\
\hline Male & 30 & 63.8 \\
\hline Female & 17 & 36.2 \\
\hline \multicolumn{3}{|l|}{ Age (years) } \\
\hline Median & 66.2 & \\
\hline Range & $44-75$ & \\
\hline \multicolumn{3}{|c|}{ ECOG performance status ${ }^{\mathrm{a}}$} \\
\hline 0 & 45 & 95.7 \\
\hline 1 & 2 & 4.3 \\
\hline \multicolumn{3}{|l|}{ Histological type } \\
\hline Differentiated & 31 & 66.0 \\
\hline Undifferentiated & 16 & 34.0 \\
\hline \multicolumn{3}{|l|}{ Tumor location } \\
\hline Upper third & 15 & 31.9 \\
\hline Middle third & 15 & 31.9 \\
\hline Lower third & 10 & 21.3 \\
\hline Entire & 7 & 14.9 \\
\hline \multicolumn{3}{|c|}{ Depth of tumor invasion ${ }^{b}$} \\
\hline $\mathrm{T} 3$ & 10 & 21.7 \\
\hline $\mathrm{T} 4$ & 37 & 78.3 \\
\hline \multicolumn{3}{|c|}{ Extent of lymph node metastasis ${ }^{b}$} \\
\hline N1 & 16 & 34.0 \\
\hline $\mathrm{N} 2$ & 25 & 53.2 \\
\hline N3 & 6 & 12.8 \\
\hline \multicolumn{3}{|l|}{ Stage ${ }^{b}$} \\
\hline IIIA & 21 & 44.7 \\
\hline IIIB & 20 & 42.5 \\
\hline IIIC & 6 & 12.8 \\
\hline
\end{tabular}

aEastern Cooperative Oncology Group; bAccording to the Japanese Classification of Gastric Carcinoma, 14th edition.

II trial. Accordingly, partial response, non-complete response/ non-progressive disease, stable disease and progressive disease were found in four $(8.5 \%), 41(87.3 \%)$, one $(2.1 \%)$, and one (2.1\%) patient, respectively. Overall response rate was $66.7 \%$ (4/6), and disease control rate was $97.9 \%$ (46/47).
Table IV. Toxicity in the phase II trial.

\begin{tabular}{lc}
\hline & $\begin{array}{c}\text { CTCAE grade } \\
\text { grade }>3(\%) \\
(\mathrm{n}=47)\end{array}$ \\
\hline $\begin{array}{l}\text { Hematological } \\
\text { Leukopenia }\end{array}$ & $3(6.4)$ \\
Neutropenia & $10(21.3)$ \\
Anemia & $4(8.5)$ \\
Non-hematological & \\
Mucositis oral & $1(2.1)$ \\
Anorexia & $2(4.3)$ \\
Nausea & $2(4.3)$ \\
Diarrhea & $1(2.1)$ \\
Gastric tumor hemorrhage & $1(2.1)$
\end{tabular}

CTCAE: The Common Terminology Criteria for Adverse Events, version 4.0

Surgical outcomes. In the phase II trial, 45 of the 47 patients underwent gastrectomy, including $17(37.8 \%)$ and 28 (62.2\%) patients who underwent distal gastrectomy and total gastrectomy, respectively. Two patients underwent exploratory laparotomy alone due to $\mathrm{T} 4 \mathrm{~b}$ (pancreas invasion) and macroscopic peritoneal dissemination in one patient, each. Among the 45 patients who underwent surgery, 44 (97.8\%) received R0 resection and one patient was determined to have positive microscopic margins (R1 resection). Postoperative morbidities were observed in eight patients (17.7\%) among those who underwent gastrectomy. Clavien-Dindo grade II complications were observed in five patients, including one, two, one, and one patient with intraabdominal abscess, chyloascites, pneumonia, and surgical site infection, respectively. Moreover, grade III morbidity was observed in three patients, including pancreatic fistula, intra-abdominal abscess, and pneumothorax in one patient each, although all patients recovered with conservative treatment.

Pathological response. Pathological response rate (grade 1b-3) was $53.3 \%$ (24/45patients: grade $1 \mathrm{~b}, \mathrm{n}=5$; grade $2, \mathrm{n}=17$; and grade $3, \mathrm{n}=2$ ). The remaining 21 patients $(46.7 \%)$ were determined to achieve grade 1a pathological response.

Postoperative chemotherapy. Postoperative adjuvant chemotherapy using S-1 was administered to 33 patients (75.0\%) after curative gastrectomy. Reasons for not administering adjuvant chemotherapy included patient rejection in eight cases and toxicities in three cases (including renal dysfunction, diarrhea, and deep vein thrombosis in one patient each). Dose adjustment and treatment interruption were required in 5 and 13 patients due to adverse effects and 

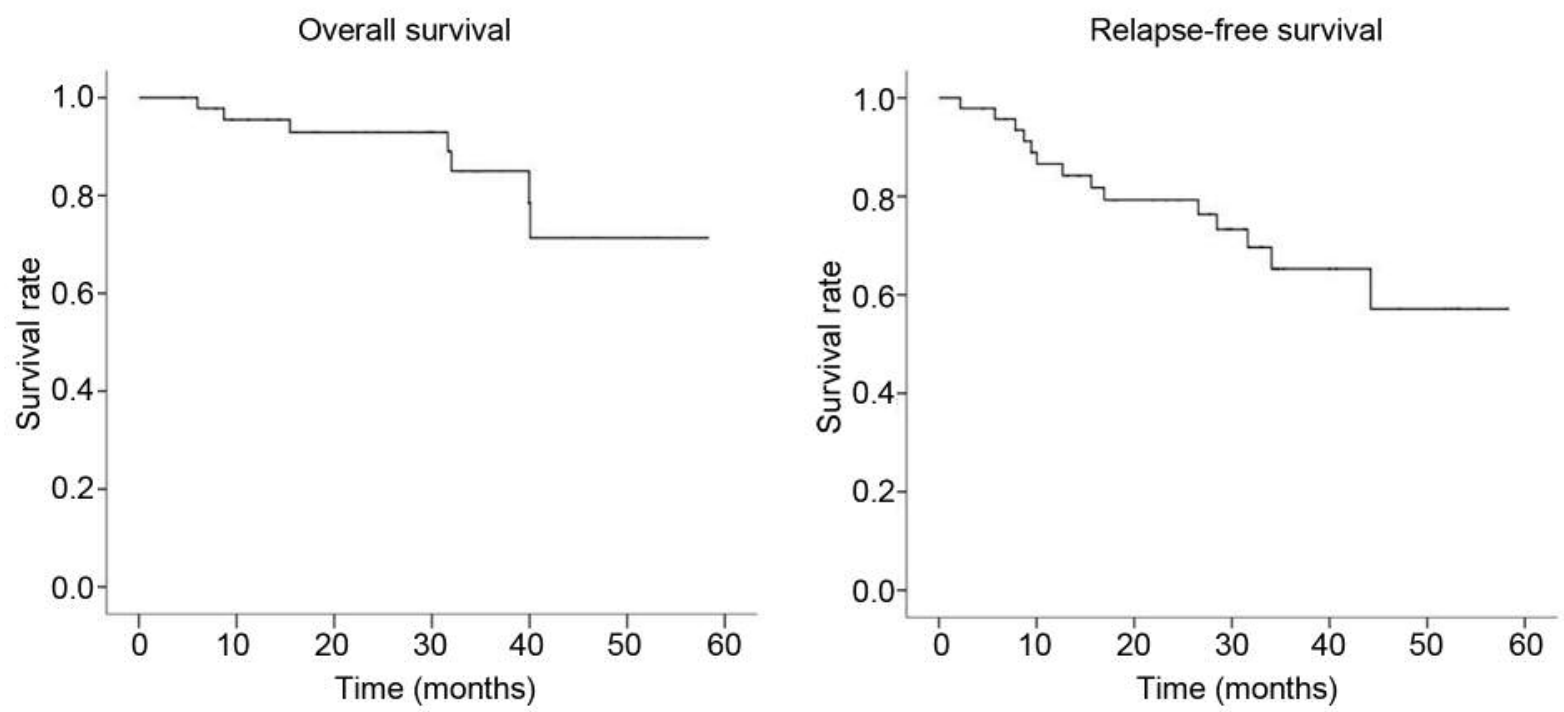

Figure 2. Kaplan-Meier analysis showing that the 3-year overall survival and relapse-free survival rates were $78.5 \%$ and $65.3 \%$, respectively.

tumor relapse, respectively. In two patients without gastrectomy due to stage IV cancer, nab-PTX was administered after exploratory laparoscopy. S-1 was also administered in one patient receiving $\mathrm{R} 1$ gastrectomy.

Survival outcomes. At the time of analysis, median followup duration was 31.6 months (range=4.6-58.2 months). A total of 31 patients were alive without relapse, whereas seven patients were alive with relapse, five patients died due to disease progression, and one patient died due to pneumonia without relapse after curative resection. Seven patients suffered from peritoneal dissemination, and five patients had lymphogenous metastases (para-aortic lymph nodes and subcarinal lymph nodes in four and one patient, respectively). Four patients developed hematogenous metastases, including three and one patient with lung and ovarian metastases, respectively.

Three-year OS and relapse-free survival (RFS) rates were $78.5 \%$ and $65.3 \%$, respectively (Figure 2). Moreover, 3-year OS and RFS rates in patients receiving curative gastrectomy were $80.2 \%$ and $69.8 \%$, respectively.

\section{Discussion}

The results of the present study suggest that RD of $50 \mathrm{mg} / \mathrm{m}^{2}$ cisplatin for NAC with biweekly DCS administration in stage III gastric cancer patients, as determined in the phase I arm, demonstrates an acceptable safety profile with acceptable survival rates in the phase II arm. NAC for gastric cancer is widely accepted in Western countries. A study showed that NAC using ECF decreases tumor size and stage and significantly improves progression-free survival and OS in operable gastric or lower esophageal adenocarcinoma patients (6). Another study suggested that NAC using 5-fluorouracil plus cisplatin significantly increases the curative resection rate, disease-free survival, and OS in patients with resectable adenocarcinoma of the lower esophagus, gastroesophageal junction, or stomach (7). Accordingly, NAC is recommended for patients with operable stage T1N0 or higher gastric cancer according to the ESMO/ESSO/ESTRO clinical practice guidelines (19) and patients with cT2 or higher gastric cancer according to the NCCN guidelines (20). However, no nationwide studies in Japan reported on the efficacy of NAC for operable gastric cancer. Therefore, well-designed randomized controlled studies are necessary to establish the efficacy of NAC in these patients.

This is the first clinical trial to evaluate NAC containing DCS for the treatment of clinical stage III gastric cancer patients. Several phase II trials conducted to evaluate DCScontaining regimens in unresectable or recurrent gastric cancer patients reported favorably high response rates (8-11, 21). Moreover, combination of NAC with DCS for locally advanced gastric cancer also demonstrated a sufficient R0 resection rate and a good pathological response $(20,22)$. However, neutropenia and febrile neutropenia were observed frequently in all trials evaluating cisplatin doses in the range of $60-70 \mathrm{mg} / \mathrm{m}^{2}(8,20-22)$ in both neoadjuvant and induction chemotherapy settings. Therefore, these DCS regimens require hospital admission (12). Moreover, this regimen is considered unsuitable for NAC because of frequent delays in scheduled surgery due to adverse events. In contrast, the present study evaluated a biweekly DCS regimen to reduce 
hematological toxicities and continue treatment with sufficient dose intensity as a potentially safe schedule at an outpatient setting. As expected, the biweekly DCS regimen was suitable as NAC, because neutropenia could be controlled on an outpatient basis with satisfactory survival outcomes. In a previous study, we reported that NAC with biweekly S-1 and docetaxel administration for locally advanced gastric cancer is associated with a low incidence of severe hematological toxicities (17). Therefore, biweekly DCS chemotherapy in the present study exhibited superior safety than that exhibited in previous studies. A previous study reported a case who suffered from thrombotic microangiopathy, which represents occlusive microvascular thrombosis, thrombocytopenia and organ damage, by the use of S-1 and cisplatin (23). Therefore, we have to pay attention to thrombotic microangiopathy when using DCS.

The target population of NAC in the present study included resectable stage III gastric cancer patients. In general, variations in the diagnostic criteria for lymph node metastasis result in diagnostic differences in clinical staging among institutions. At our institution, lymph node metastasis was defined as the presence of lymph nodes with a short diameter larger than $8 \mathrm{~mm}$ as assessed by computed tomography, although the RECIST criteria set the lymph node diameter cutoff to $15 \mathrm{~mm}$. Therefore, the ratio of patients without measurable lesions was high $(87.3 \%)$ in the present study; subsequently, the evaluation of tumors in these patients was frequently estimated as non$\mathrm{CR} /$ non-PD according to the RECIST criteria.

In the present study, the $\mathrm{R} 0$ resection rate $(93.6 \%)$ was similar to those reported in previous studies $(20,22)$. Despite diagnostic laparoscopy performed prior to NAC, some patients progressed to an unresectable disease stage. The possibility of undetected peritoneal dissemination by diagnostic laparoscopy and the advancement of tumors refractory to the DCS triplet regimen in a subset of population should thus be considered.

Previous studies reported that pathological response was superior to the RECIST criteria for evaluating the efficacy of NAC and that pathological response predicted long-term outcomes more clearly $(24,25)$. Furthermore, another study reported that pathological response was a prognostic factor in advanced gastric cancer patients receiving the DCS regimen in a NAC setting (26). Thus, further investigation is necessary to evaluate prognostic factors for long-term survival in this NAC setting after a sufficient follow-up duration. Moreover, the pathological response rate, which was $53.3 \%$ in the present study, was $65.9 \%$ in another study (18) that comprised two courses of preoperative chemotherapy with S-1 (40 mg/m $\mathrm{m}^{2}$ b.i.d) on days $1-14$ and docetaxel $\left(60 \mathrm{mg} / \mathrm{m}^{2}\right)$ plus cisplatin $\left(60 \mathrm{mg} / \mathrm{m}^{2}\right)$ on days 8 every three weeks (18); that study also reported 3-year OS and PFS rates of $89.7 \%$ and $85.9 \%$, respectively, with a high incidence of hematological toxicities. Although the pathological response rate was low in the present study, survival periods of patients who underwent gastrectomy were comparable to those of patients who participated in the previous NAC study. Therefore, biweekly DCS is a favorable regimen as NAC for stage III gastric cancer patients.

In the present study, postoperative complications developed in eight patients $(17.8 \%)$; the rate and degree of postoperative complications were also lower than those reported in previous studies on NAC for advanced gastric cancer $(27,28)$. Therefore, this biweekly DCS regimen is suitable as NAC with lower postoperative morbidity.

In conclusion, the findings of the present phase I/II trial revealed that the RD for cisplatin is $50 \mathrm{mg} / \mathrm{m}^{2} /$ day, as part of a biweekly DCS combination as NAC for stage III gastric cancer patients. This regimen might be suitable for use on an outpatient basis as an effective, safe, and acceptable treatment protocol. Further investigation is necessary to assess long-term survival rates for confirmation of the efficacy of biweekly DCS as NAC for stage III gastric cancer patients.

\section{Conflicts of Interest}

The Authors declare that they have no conflict of interest regarding this study.

\section{References}

1 Jemal A, Siegel R, Xu J and Ward E: Cancer statistics, 2010. CA Cancer J Clin 61: 133-134, 2011.

2 Songun I, Putter H, Kranenbarg EM Sasako M and van de Velde CJ: Surgical treatment of gastric cancer: 15-year follow-up results of the randomised nationwide Dutch D1D2 trial. Lancet Oncol 11: 439-449, 2010.

3 Sasako M, Sano T, Yamamoto S, Kurokawa Y, Nashimoto A, Kurita A, Hiratsuka M, Tsujinaka T, Kinoshita T, Arai K and Yamamura Y: D2 lymphadenectomy alone with or para-aortic nodal dissection for gastric cancer. N Engl J Med 359: 453-462, 2008.

4 Sakuramoto S, Sasako M, Yamaguchi T, Kinoshita T, Fujii M, Nashimoto A, Furukawa H, Nakajima T, Ohashi Y, Imamura H, Higashino M, Yamamura Y, Kurita A and Arai K; ACTS-GC Group: Adjuvant chemotherapy for gastric cancer with S-1, an oral fluoropyrimidine. N Engl J Med 357: 1810-1820, 2007.

5 Sasako M, Sakuramoto S, Katai H, Kinoshita T, Furukawa H, Yamaguchi T, Nashimto A, Fujii M, Nakajima T and Ohashi Y: Five-year outcomes of a randomized phase III trial comparing adjuvant chemotherapy with S-1 versus surgery alone in stage II or III gastric cancer. J Clin Oncol 29: 4387-4393, 2011.

6 Cunningham D, Allum WH, Stenning SP Thompson JN, Van de Velde CJ, Nicolson M, Scarffe JH, Lofts FJ, Falk SJ, Iveson TJ and Smith DB: Perioperative chemotherapy versus surgery alone for resectable gastroesophageal cancer. N Engl J Med 355: 11-20, 2006.

7 Ychou M, Boige V, Pignon JP Conroy T, Bouché O, Lebreton G, Ducourtieux M, Bedenne L, Fabre JM, Saint-Aubert B and Genève J: Perioperative chemotherapy compared with surgery alone for resectable gastroesophageal adenocarcinoma: an FNCLCC and FFCD multicenter phase III trial. J Clin Oncol 29: 1715-1721, 2011. 
8 Sato Y, Takayama T, Sagawa T, Takahashi Y, Ohnuma H, Okubo S, Shintani N, Tanaka S, Kida M, Sato Y, Ohta H, Miyanishi K, Sato T, Takimoto R, Kobune M, Yamaguchi K, Hirata K, Niitsu Y and Kato J: Phase II study of S-1, docetaxel and cisplatin combination chemotherapy in patients with unresectable metastatic gastric cancer. Cancer Chemother Pharmacol 66: 721-728, 2010

9 Takayama T, Sato Y, Sagawa T, Okamoto T, Nagashima H, Takahashi Y, Ohnuma H, Kuroiwa G, Miyanishi K, Takimoto R, Matsunaga T, Kato J, Yamaguchi K, Hirata K and Niitsu Y: Phase I study of S-1, docetaxel and cisplatin combination chemotherapy in patients with unresectable metastatic gastric cancer. Br J Cancer 97: 851-856, 2007.

10 Nakayama N, Koizumi W, Sasaki T, Higuchi K, Tanabe S, Nishimura K, Katada C, Nakatani K, Takagi S and Saigenji K: A multicenter, phase I dose-escalating study of docetaxel, cisplatin and S-1 for advanced gastric cancer (KDOG0601). Oncology 75: 1-7, 2008.

11 Hironaka S, Yamazaki K, Taku K, Yokota T, Shitara K, Kojima T, Ueda S, Machida N, Muro K and Boku N: Phase I study of docetaxel, cisplatin and S-1 in patients with advanced gastric cancer. Jpn J Clin Oncol 40: 1014-1020, 2010.

12 Fushida S, Fujimura T, Oyama K, Yagi Y, Kinoshita J and Ohta T: Feasibility and efficacy of preoperative chemotherapy with docetaxel, cisplatin and S-1 in gastric cancer patients with paraaortic lymph node metastases. Anticancer Drugs 20: 752-756, 2009.

13 Japanese Gastric Cancer Association: Japanese classification of gastric carcinoma: 3rd English edition. Gastric Cancer 14: 101112, 2011.

14 Kimura Y, Yano H, Imamura H, Fujitani K, Imano M, Tokunaga Y, Matsuoka M, Kurokawa Y, Shimokawa T, Takiuchi H, Tsujinaka $\mathrm{T}$ and Furukawa H: A phase I study of triplet combination chemotherapy of paclitaxel, cisplatin and S-1 in patients with advanced gastric cancer. Jpn J Clin Oncol 43: 125-131, 2013.

15 Common Terminology Criteria for Adverse Events (CTCAE) v4.0, National Cancer Institute. https://ctep.cancer.gov/ protocolDevelopment/electronic_applications/ctc.htm\#ctc_40, April. 6, 2018.

16 Eisenhauer EA, Therasse P, Bogaerts J, Schwartz LH, Sargent D, Ford R, Dancey J, Arbuck S, Gwyther S, Mooney M, Rubinstein L, Shankar L, Dodd L, Kaplan R, Lacombe D and Verweij J: New response evaluation criteria in solid tumours: revised RECIST guideline (version 1.1). Eur J Cancer 45: 228247, 2009

17 Kosaka T, Akiyama H, Makino H, Takagawa R, Kimura J, Ono $\mathrm{H}$, Kunisaki C and Endo I: Preoperative S-1 and docetaxel combination chemotherapy in patients with locally advanced gastric cancer. Cancer Chemother Pharmacol 73: 281-285, 2014.

18 Hirakawa M, Sato Y, Ohnuma H, Takayama T, Sagawa T, Nobuoka T, Harada K, Miyamoto H, Sato Y, Takahashi Y, Katsuki S, Hirayama M, Takahashi M, Ono M, Maeda M, Takada K, Hayashi T, Sato T, Miyanishi K, Takimoto R, Kobune $\mathrm{M}$, Hirata $\mathrm{K}$ and Kato $\mathrm{J}$ : A phase II study of neoadjuvant combination chemotherapy with docetaxel, cisplatin, and S-1 for locally advanced resectable gastric cancer: nucleotide excision repair (NER) as potential chemoresistance marker. Cancer Chemother Pharmacol 71: 789-797, 2013.

19 Smyth EC, Verheij M, Allum W, Cunningham D, Cervantes A and Arnold D: Gastric cancer: ESMO Clinical Practice Guidelines for diagnosis, treatment and follow-up. Ann Oncol 27: v38-v49, 2016.
20 Ajani JA, D'Amico TA, Almhanna K, Bentrem DJ, Chao J, Das P, Denlinger CS, Fanta P, Farjah F, Fuchs CS, Gerdes H, Gibson M, Glasgow RE, Hayman JA, Hochwald S, Hofstetter WL, Ilson DH, Jaroszewski D, Johung KL, Keswani RN, Kleinberg LR, Korn WM, Leong S, Linn C, Lockhart AC, Ly QP, Mulcahy MF, Orringer MB, Perry KA, Poultsides GA, Scott WJ, Strong VE, Washington MK, Weksler B, Willett CG, Wright CD, Zelman D, McMillian N and Sundar H: Gastric Cancer, Version 3.2016, NCCN Clinical Practice Guidelines in Oncology. J Natl Compr Canc Netw 14: 1286-1312, 2016.

21 Koizumi W, Nakayama N, Tanabe S, Sasaki T, Higuchi K, Nishimura K, Takagi S, Azuma M, Ae T, Ishido K, Nakatani K, Naruke A and Katada C: A multicenter phase II study of combined chemotherapy with docetaxel, cisplatin and S-1 in patients with unresectable or recurrent gastric cancer (KDOG 0601). Cancer Chemother Pharmacol 69: 407-413, 2012.

22 Aoyama T, Nishikawa K, Fujitani K, Tanabe K, Ito S, Matsui T, Miki A, Nemoto H, Sakamaki K, Fukunaga T, Kimura Y, Hirabayashi $\mathrm{N}$ and Yoshikawa T: Early results of a randomized two-by-two factorial phase II trial comparing neoadjuvant chemotherapy with two and four courses of cisplatin/S-1 and docetaxel/cisplatin/S-1 as neoadjuvant chemotherapy for locally advanced gastric cancer. Ann Oncol 28: 1876-1881, 2017.

23 Muto J, Kishimoto H, Kaizuka Y, Kinjo M, Higashi H and Kishihara F: Thrombotic microangiopathy following chemotherapy with S-1 and cisplatin in a patient with gastric cancer: A case report. In Vivo 31: 439-441, 2017.

24 Kurokawa Y, Shibata T, Ando N, Seki S, Mukaida H and Fukuda $\mathrm{H}$ : Which is the optimal response criteria for evaluating preoperative treatment in esophageal cancer: RECIST or Histology? Ann Surg Oncol 20: 3009-3014, 2013.

25 Kurokawa Y, Shibata T, Sasako M, Sano T, Tsuburaya A, Iwasaki $\mathrm{Y}$ and Fukuda $\mathrm{H}$ : Validity of response assessment criteria in neoadjuvant chemotherapy for gastric cancer (JCOG0507-A). Gastric Cancer 17: 514-521, 2014.

26 Sasaki K, Onodera S, Otsuka K, Satomura H, Kurayama E, Kubo T, Takahashi M, Ito J, Nakajima M, Yamaguchi $\mathrm{S}$ and Miyachi K: Validity of neoadmuvant chemotherapy with docetaxel, cisplatin, and S-1 for resectable locally advanced gastric cancer. Med Oncol 34: 139, 2017.

27 Iwasaki Y, Sasako M, Yamamoto S, Nakamura K, Sano T, Katai $\mathrm{H}$, Tsujinaka T, Nashimoto A, Fukushima $\mathrm{N}$ and Tsuburaya A: Gastric cancer surgical study group of Japan clinical oncology group. Phase II study of preoperative chemotherapy with S-1 and cisplatin followed by gastrectomy for clinically resectable type 4 and large type 3 gastric cancers (JCOG0210). J Surg Oncol 107: 741-745, 2013.

28 Yoshikawa T, Sasako M, Yamamoto S, Sano T, Imamura H, Fujitani K, Oshita H, Ito S, Kawashima Y and Fukushima N: Phase II study of neoadjuvant chemotherapy and extended surgery for locally advanced gastric cancer. Br J Surg 96: 1015-1022, 2009.
Received August 5, 2018

Revised August 30, 2018

Accepted September 6, 2018 